\title{
Charge Glass in Two-Dimensional Arrays of Capacitively Coupled Grains with Random Offset Charges
}

\author{
Enzo Granato \\ Laboratório Associado de Sensores e Materiais, Instituto Nacional de Pesquisas Espaciais, 12201 São José dos Campos, SP, Brazil \\ J. M. Kosterlitz \\ Department of Physics, Brown University, Providence, Rhode Island 02912 \\ (Received 3 August 1998)
}

\begin{abstract}
We study the effect of random offset charges in the insulator to conductor transition in systems of capacitively coupled grains, as realized in two-dimensional arrays of ultrasmall Josephson junctions. In the presence of disorder, the conductive transition and charge ordering at nonzero gate voltages are both destroyed for any degree of disorder at finite temperatures in the thermodynamic limit, but crossover effects will dominate at finite length scales. The conductance is linear and thermally activated but nonlinear behavior sets in at a crossover voltage which decreases as temperature decreases. For large disorder, the results are supported by Monte Carlo dynamics simulations of a Coulomb gas with offset charges and are consistent with the thermally activated behavior found in recent experiments. [S0031-9007(98) 07499-7]
\end{abstract}

PACS numbers: 64.70.Pf, 73.40.Gk, 73.40.Rw

Two-dimensional arrays of Josephson junctions where the charging energy of the grains is much larger than the Josephson coupling energy are interesting systems where collective charging effects can be studied experimentally in great detail [1-4]. In these systems, the net charges in the grains have a long range logarithmic interaction when the junction capacitance $C$ is small enough. The charges play the same role as vortices in the resistive behavior of the array, when capacitive effects can be neglected. This allows for the possibility of a Kosterlitz-Thouless (KT) charge unbinding transition corresponding to an insulating to conducting transition at finite temperature [1]. Below the critical temperature $T_{c}$, neutral charge pairs would be present in the insulating phase while above $T_{c}$, screening of the electrostatic potential gives rise to free isolated charges and a conducting phase. Experimentally, a transition has in fact been observed in the conductance of small capacitance arrays of normal and superconducting grains $[1-3]$ at an apparent temperature consistent with the estimate based on logarithmically interacting charges. Recently, however, a closer analysis of the experimental data of different groups revealed that the onset of finite conductance at finite temperatures could be described just as well by thermal activation of free charges $[2,3]$ with an activation energy $E_{a} \approx \frac{1}{4} E_{c}$ when the grains are in the normal state and $E_{a} \approx \frac{1}{4} E_{c}+\Delta$ for superconducting grains, where $2 \Delta$ is the superconducting energy gap at zero temperature. This interpretation neglects the logarithmic interaction between charges expected for an ideal array $[1,4]$ and suggests in turn that, in the experimental systems, the interaction is screened by some unknown mechanism and is essentially short ranged. Although disorder effects, in the form of random offset charges which may be trapped near the grain-substrate interface [1], could be a possible explanation for these results, an understanding of how differently prepared systems, and therefore different degrees of disorder, can still lead to the same activated behavior of the linear conductance is still lacking. In particular, measurable nonlinear effects resulting from disorder have not been considered so far.

In this Letter, we consider the effects of random offset charges on the charge-anticharge unbinding transition in an otherwise perfect two-dimensional array of capacitively coupled normal or superconducting grains in the classical regime of large charging energy $E_{c}$ and finite temperature $T$. We show that, in the presence of offset charges, the unbinding transition and charge ordering at nonzero gate voltages are both destroyed for any degree of disorder $\sigma$ in the thermodynamic limit, consistent with the common behavior of different experimental arrays. The conductance is linear and thermally activated, but crossover effects will dominate at length scales smaller than a disorder and temperature dependent length, $\xi_{\sigma, T}=T^{-\nu} f\left(\sigma T^{-\nu}\right)$, where $\nu$ is the thermal critical exponent characterizing the $T=0$ charge glass transition. Nonlinear behavior sets in at a characteristic voltage $V_{c} \sim T / \xi_{\sigma, T}$. For larger disorder, the results are supported by a Monte Carlo simulation of the nonlinear conductance leading to $V_{c} \sim T^{1+\nu}$ with a very rough estimate $\nu \sim 1.7$.

The electrostatic energy of the net mobile charges $Q_{i}=$ $e^{*} n_{i}$, multiples of an elementary charge $e^{*}$, located at the sites $i$ of an array is given by [1,5]

$$
E=\frac{1}{2} \sum_{i, j}\left(Q_{i}+q_{i}\right) C_{i j}^{-1}\left(Q_{i}+q_{i}\right),
$$

where $e^{*}=e$, the electron charge, for a normal grain and $e^{*}=2 e$ for a superconducting grain. $C_{i j}$ is the capacitance matrix and $q_{i}$ represents a net offset charge in a grain induced by charged impurities trapped near the grainsubstrate interface $[1,6]$. The equilibrium properties in the 
classical limit are described by the partition function $Z=$ $\sum_{\left\{n_{i}\right\}} e^{-E / k T}$, where the sum is over all integers $n_{i}$ and the offset charges $q_{i}$ act as quenched disorder with a nonzero uncorrelated component. We also assume that tunneling of charges between the grains provides the dynamics to equilibrate the charge distribution and the junction dissipation is sufficiently small [1]. Since an offset charge equal to an integer number of $e^{*}$ can be compensated by shifting $n_{i} \rightarrow n_{i}-m$, one can consider offset charges with a probability distribution of width $\sigma<e^{*}$ peaked at zero. In general, the elements of the inverse capacitance matrix $C_{i j}^{-1}$ fall off exponentially for $r_{i j}>\Lambda$, where $\Lambda \sim$ $\sqrt{C / C_{g}}$ is a screening length, $C$ is the capacitance between neighboring grains, and $C_{g}$ is the capacitance to the ground. We consider the regime $r / \Lambda \ll 1$, where [5] $C_{i j}^{-1}=-\frac{1}{2 \pi C} \log r_{i j}-1 / 4 C$. Under these conditions, the interaction energy above describes a two-dimensional Coulomb gas of logarithmically interacting charges $Q_{i}$ in a background of quenched random charges $q_{i}$. This problem has been considered before in relation to the effects of geometrical disorder in classical superconducting arrays in a magnetic field where $Q_{i}$ represents vortices and $q_{i}$ a random flux [7] and in the gauge-glass model [8] of disordered superconductors where the $q_{i}$ are random but correlated.

In the absence of offset charges, $q_{i}=0$, a chargeanticharge unbinding transition in the KT universality class has been predicted [1] at a finite temperature $k T_{c} \approx e^{* 2} / 8 \pi C$. However, in the presence of offset charges with a nonzero component of uncorrelated disorder, $\left\langle q_{i} q_{j}\right\rangle=\sigma^{2} \delta_{i j}$, the behavior is drastically changed as bound charge-anticharge pairs are unstable at a sufficiently large length scale $\xi$ determined by the degree of disorder. In fact, the net charge fluctuation due to random charges in a domain of area $\pi L^{2}$ will neutralize a test charge $Q=e^{*}$ when $L=\xi_{\sigma}=e^{*} / \sqrt{\pi} \sigma$. For length scales larger than $\xi_{\sigma}$, charges are unbound and will contribute to the linear conductance $G$ provided that charges are mobile. This shows that even for small $\sigma$, disorder is a strongly relevant perturbation.

In general, there will also be a contribution from correlated disorder. However, this is either irrelevant at the fixed point of the pure system or at most marginal for sufficiently small disorder. To lowest order in the wave vector $k$, the moments of disorder distribution are given by $\left\langle q_{k}\right\rangle=0$ and $\left\langle q_{k} q_{-k}\right\rangle=\sigma^{2}+\sigma_{2}^{2} k^{2}+O\left(k^{4}\right)$. The first term corresponds to uncorrelated randomness as discussed above and the second term to offset charges correlated as random dipoles $p=q\left|r_{i}-r_{j}\right|$, where $r_{i}$ and $r_{j}$ are neighboring sites, since $\left\langle p_{k} p_{-k}\right\rangle=\sigma_{2}^{2} k^{2}$. Higher order terms correspond to multipole correlations. Using standard methods to convert the partition function of Eq. (1) to a sine-Gordon model [9] and the replica trick to average the free energy over disorder, we obtain an effective reduced Hamiltonian in terms of $n$ replicas

$$
H / k T=\frac{1}{2} \int d^{2} r\left[\frac{1}{K} \sum_{\alpha}\left(\nabla \varphi^{\alpha}\right)^{2}+\left(\frac{2 \pi \sigma}{e^{*}}\right)^{2} \sum_{\alpha, \beta} \varphi_{r}^{\alpha} \varphi_{r}^{\beta}+\left(\frac{2 \pi \sigma_{2}}{e^{*}}\right)^{2} \sum_{\alpha, \beta} \nabla \varphi^{\alpha} \nabla \varphi^{\beta}-y \sum_{\alpha} \cos \left(2 \pi \varphi_{r}^{\alpha}\right)\right],
$$

where $K=E_{c} / 2 \pi^{2} k T$. Higher order gradient terms have been neglected. In the absence of disorder this model has a massless phase for $\pi K>2$ when the charge fugacity $y$ is irrelevant corresponding to an insulating phase. For $\pi K<2, y$ is relevant leading to a conducting phase. From renormalization-group arguments, the relevance of the disorder perturbation on the massless phase can now be determined from the eigenvalue $\lambda=d-x$ where $2 x$ is the correlation function exponent evaluated at the unperturbed fixed point. For uncorrelated disorder, $\sigma \neq 0$, it is sufficient to consider the diagonal contribution $\alpha=\beta$. This has an eigenvalue $\lambda=2$ in the massless phase since $x=(d-2)=0$ in two dimensions, which corresponds to a scaling behavior $\sigma^{\prime 2}=\sigma^{2} b^{2}$ under a change of lattice spacing by a factor of $b$, in agreement with the domain argument given above leading to a characteristic disorder length $\xi_{\sigma} \propto 1 / \sigma$. For correlated disorder alone, $\sigma_{2} \neq 0$, the contribution from the diagonal term $\sum_{\alpha} \nabla \varphi_{\alpha} \nabla \varphi_{\alpha}$ just renormalizes the value of $K$ but the off-diagonal perturbation $\sum_{\alpha \neq \beta} \nabla \varphi_{\alpha} \nabla \varphi_{\beta}$ is marginal, $\lambda=0$. The higher order gradient terms in the Hamiltonian are then irrelevant.

The disorder dependent length $\xi_{\sigma}$ is the scale up to which the charges $Q_{i}$ are mobile. Beyond this scale, they are neutralized by the random background of quenched charges $q_{i}$. At a scale $L>\xi_{\sigma}$, the system is better described as a set of pinned charges interacting by a short range potential, and we expect that it behaves similarly to a charge glass. Since these charges play the same role as vortices in a two-dimensional superconductor, one expects that glass order occurs at $T=0$ only, with a thermal correlation length $\xi_{T} \propto T^{-\nu}$, where $\nu$ is a thermal critical exponent associated with the $T=0$ fixed point [8]. Assuming that the model of Eq. (1) is in the same universality class, we expect that $\nu \gtrsim 2$. Standard crossover scaling leads to a correlation length $\xi_{\sigma, T}=$ $T^{-\nu} f\left(\sigma T^{-\nu}\right)$ where the scaling function $f(x)$ is a function of the dimensionless ratio $x=\xi_{T} / \xi_{\sigma}$ with $f(x) \sim 1$ for $x \gg x_{c}$ and $f(x) \sim 1 / x$ for $x \ll x_{c}$ so that $\xi_{\sigma, T} \propto 1 / \sigma$ when $\sigma T^{-\nu}<x_{c}$ and $\xi_{\sigma, T} \propto T^{-\nu}$ for $\sigma T^{-\nu}>x_{c}$. For an infinite system, the predicted insulating-conducting transition in the absence of disorder is destroyed by any finite density of uncorrelated offset charges but crossover effects may lead to an apparent transition if $\xi_{\sigma, T}$ is larger than the system size. Note that the above arguments are not strictly correct in the limit $\sigma=0$ or $T=0$ as it does not allow for the possibility of a transition to a true insulating phase of bound pairs of charges at finite 
$T$ when $\sigma=0$ or for quantum tunneling [3] at $T=0$. In principle, such effects could be incorporated in the crossover scaling analysis at the expense of some major extra complications, but experiments [1-3] indicate that there is a range of temperatures over which purely classical behavior is observed and that behavior characteristic of the absence of disorder is never observed. The crossover scaling analysis outlined above should be relevant to experimental systems at temperatures above the crossover temperature where quantum tunneling dominates [3].

In addition to $\xi$, another length scale, $\xi_{V}$, is set by an applied finite voltage $V$ across the system [10]. The additional contribution to the energy has the form $\sum_{i} Q_{i} E x_{i}$ for a uniform electric field $E$ in the $x$ direction. Thermal fluctuations alone, of typical energy $k T$, lead to a characteristic length $\xi_{V} \sim k T / e^{*} E$ over which single charge motion is possible. For $\xi_{V}>\xi$, when charges are unbound, this leads to a linear response to the applied voltage and a finite conductance limited by the tunneling rate across each junction in the array. For $\xi_{V}<$ $\xi$, the contribution from the applied voltage must be balanced against the interaction energy with other charges leading to nonlinear behavior. A crossover from linear to nonlinear behavior occurs when these two lengths are comparable, i.e., at a characteristic voltage $V_{c} \sim$ $T / \xi_{\sigma, T}=T^{\nu+1} g\left(\sigma T^{-\nu}\right)$, where $g(x)=1 / f(x)$.

The finite correlation length $\xi$ in the presence of disorder also determines the behavior of the activation energy in the regime of linear conductance. For vanishing voltage, the current arises from thermal dissociation of the most weakly bound charge pairs. For normal metals, the energy necessary to unbind a typical pair is $E=\frac{E_{c}}{\pi} \log (\xi / a)+E_{o}$, where $E_{o} \simeq E_{c} / 2$ is the core energy of a pair with one lattice spacing separation. For sufficiently large disorder such that $\xi \sim a$, the conductance should then have an Arrhenius behavior with an activation energy given [2] by $E_{a}=E_{o} / 2$. However, for moderate disorder the activation energy has a temperature and disorder dependent logarithmic correction $\log \left(\xi_{\sigma, T} / a\right)$. In the pure case where $\sigma=0$ and $\xi_{\sigma, T} \rightarrow \infty$, this implies an infinite activation energy and the linear conductance vanishes consistent with $V_{c} \sim T / \xi_{\sigma, T} \rightarrow 0$. For superconducting grains, the arguments above still apply except that an additional energy [2] equal to the superconducting energy gap $E_{g}$ is required to create quasiparticles which allow charges on the grains to tunnel to neighboring grains, in the absence of Josephson tunneling.

In the presence of a gate voltage $V_{g}$ on each grain, there will be an induced charge $C_{g} V_{g}$ on each grain acting as a frustration parameter [1] $f=C_{g} V_{g} / e^{*}$ analogous to the flux quanta per plaquette in Josephson junction arrays in a magnetic field. The properties of the system should be periodic in $f$ with period 1 . In particular, the Coulomb gap width oscillates as a function of $V_{g}$ with period $e^{*} / C_{g}$, often used as an estimate of $C_{g}$. For fractional values of $f$ the ground state is a charged ordered state with an average number $f$ of elementary charges per grain. For rational $f=p / q$, this leads to a ground state with a unit cell of size $q \times q$ and a discrete degenerate ground state [11]. A finite temperature transition is expected from the charge ordered phase to a disordered phase in addition to the charge-anticharge unbinding transition. In the presence of disorder, the behavior at integer $f$ will be the same as $f=0$, i.e., at any finite temperature the interacting charge system is disordered and the conductance is finite. This behavior should persist for fractional values of $f$ since, in addition to the discrete symmetry excitations such as domain walls, thermally excited net charges interacting logarithmically are also present which will be screened by disorder at length scales larger than $\xi$ leading to a linear thermally activated conductance. However, one still may question if charge order remains stable in the presence of disorder. It is sufficient to consider the case $f=1 / 2$ since the ground states for $f<1 / 2$ are less stable [11]. At $f=1 / 2$ and in the absence of disorder, it is known that the ground state [11] consists of $2 \times$ 2 unit cells with an antiferromagnetic arrangement of $n_{i}-f= \pm 1 / 2$ net fractional charges, in units of the elementary charge $e^{*}$. The lowest energy excitations are domain walls separating the two degenerate ground states, $\pm 1 / 4$ charges at domain wall corners, and unit excess charges interacting logarithmically. In a coarse-grained description, the variables in a unit cell can be replaced by Ising variables $S_{r}= \pm 1$, which describe the charge ordered state and charges $Q_{R}=\sum S_{r} / 4$ averaged over the unit cell [12]. This leads to an effective Hamiltonian [7]

$$
H / k T=L_{1} \sum_{\left\langle r, r^{\prime}\right\rangle} S_{r} S_{r^{\prime}}+2 \pi^{2} K \sum_{R, R^{\prime}} Q_{R} G\left(R-R^{\prime}\right) Q_{R^{\prime}}+L_{2} \sum_{r} S_{r} q_{r}+4 \pi^{2} K \sum_{r R} Q_{R} G(R-r) q_{r},
$$

where $G(r)=\log (r / a)+\pi / 2$. The Ising variables $S_{r}$ are coupled antiferromagnetically by a nearest neighbor interaction $L_{1}$ and to a random field proportional to $q_{r}$. The excess charges $Q_{R}$ are still coupled to the random offset charges $q_{r}$ and will therefore lead to finite conductance as discussed before. Since the offset charges coupled as random fields to the Ising variables, charge order will be destroyed even for small disorder. There will be, however, a characteristic length scale [13] $\xi_{\sigma}^{\prime} \sim \exp \left(c / \sigma^{2}\right)$, beyond which random offset charges destroy the charge ordered state. For sufficiently small $\sigma, \xi_{\sigma}^{\prime}>\xi_{\sigma}$ and there can be an intermediate length scale where charge order remains but excess charge is effectively unbound. Similar results should apply for $f<1 / 2$ and we expect that disorder destroys charge ordering for any finite gate voltage.

We have studied the linear and nonlinear conductance of the Coulomb gas in Eq. (1) numerically using classical Monte Carlo nonequilibrium dynamics [8], regarding the Monte Carlo time as real time. This should be a reasonable 
approximation in the overdamped limit and when quantum effects can be ignored. It also assumes that the tunneling rate which provides the equilibrium charge distribution satisfies the global rule [14] when it depends on the energy difference of the whole array. In the simulations, a Monte Carlo step consists of adding a dipole of unit charges and unit length to a nearest neighbor charge pair, $\left(n_{i}, n_{j}\right)$, corresponding to a tunneling of the positive or negative charge by a unit of length. The external electric field biases the added dipole, leading to a current as the net flow of charge is in the direction of the electric field. The unit of time corresponds to a complete Monte Carlo pass through the system. The linear conductance $G_{L}=\lim _{E \rightarrow 0} J / E$ is obtained from the equilibrium current fluctuations, without imposing a voltage bias, from the fluctuation-dissipation relation $G_{L}=\frac{1}{2 k T} \int d t\langle I(0) I(t)\rangle$.

Figures 1(a) and 1(b) show the behavior of the linear conductance $G_{L}$ in the absence of disorder, $\sigma=0$, and in the presence of a (large) random Gaussian distribution of offset charges with a standard deviation $\sigma=0.5$. Since large disorder leads to small $\xi$, we can use a small system size $L \times L$ with $L=16$. When $\sigma=0$, the conductance appears to vanish at $T_{c} \approx 1.4$ for $f=0$ and $\approx 0.8$ for $f=1 / 2$, corresponding to the critical temperature for the conductive transition for zero and nonzero gate voltages. However, for $\sigma=0.5$, the conductance remains finite much below the critical temperature for the pure system. In fact, as indicated in the figure, the data in the low temperature range can be reasonably fitted to the Arrhenius activated form $G=G_{o} e^{-E_{a} / k T}$ in agreement with a disordered phase. Moreover, the calculation of $G_{L}$ from current fluctuations ensures that the activated behavior is not an artifact of a voltage bias since it is obtained without an external electric field. In experiments, however, a volt-
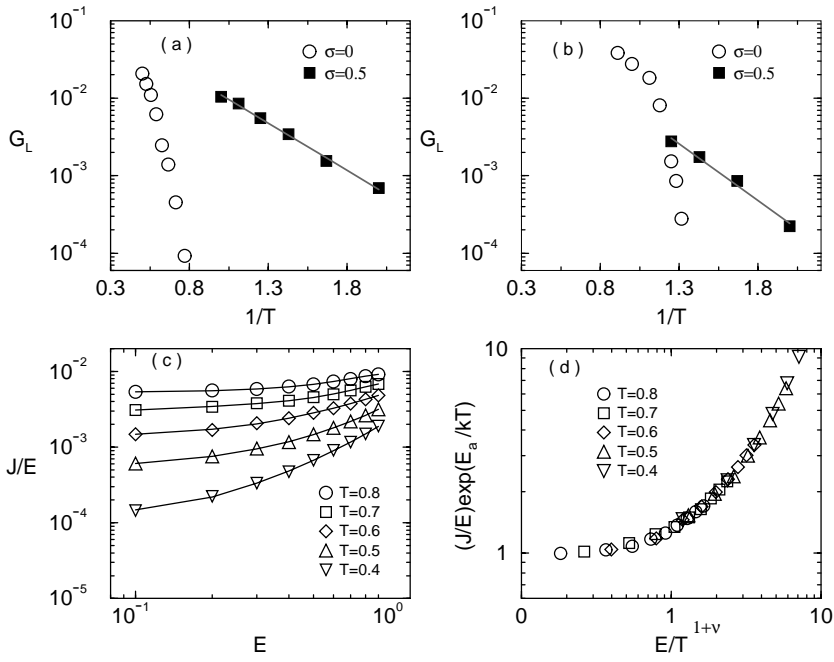

FIG. 1. (a) Linear conductance $G_{L}$ with zero gate voltage $(f=0)$ and (b) with nonzero gate voltage $(f=1 / 2)$. The straight lines indicate an Arrhenius behavior. Temperature $T$ is measured units of $E_{c} / 2 \pi^{2}$. (c) Nonlinear conductance $J / E$ as a function of temperature, for $\sigma=0.5$ and $(f=0)$. (d) Scaling plot of the nonlinear conductance in (c). age bias is often used and nonlinear effects should be taken into account. In Fig. 1(c) we show the behavior of the nonlinear conductance as a function of the applied electric field and temperature for $\sigma=0.5$ and $f=0$. For small $E, J / E$ tends to the constant finite value of Fig. 1(a) which decreases with temperature. For the highest temperature, $T=0.8$, the range of $E$ in which this behavior is apparent is more pronounced. For increasing $E$ it clearly crosses over to a nonlinear behavior in agreement with the analysis leading to a crossover voltage $V_{c}$ which is both temperature and disorder dependent. The nonlinear conductance can be cast into a scaling form [8], $J / E G_{L}=F\left(E / E_{c}\right)$, where $F$ is some scaling function with $F(0)=1$. For large disorder we have $E_{c} \sim T^{\nu+1}$, and a scaling plot allows an estimate of $\nu$. This is shown in Fig. 1(d) where a reasonable data collapse is obtained by adjusting $E_{a}$ and $\nu$ for the largest temperatures, giving $\nu \sim 1.7$, in rough agreement with the $T=0$ stiffness exponent for gauge glass models [8]. This implies that the characteristic voltage $V_{c}$ where nonlinearities set in should exhibit a nontrivial power law $V_{c} \sim T^{\nu+1}$ and should be accessible experimentally.

This work was supported by FAPESP, Proc. No. 97/07250-8 (E. G.) and Proc. No. 97/9188-8 (J. M. K.), and by a joint CNPq-NSF grant. We acknowledge the support from ICTP, where part of the work was done.

[1] J.E. Mooij, B. J. van Wees, L.J. Geerligs, M. Peters, R. Fazio, and G. Schön, Phys. Rev. Lett. 65, 645 (1990).

[2] T.S. Tighe, M. T. Tuominen, J. M. Hergenrother, and M. Tinkham, Phys. Rev. B 47, 1145 (1993).

[3] P. Delsing, C. D. Chen, D. B. Haviland, Y. Harada, and T. Claeson, Phys. Rev. B 50, 3959 (1994); P. Delsing, C. D. Chen, D. B. Haviland, T. Bergesten, and T. Claeson, cond-mat/9805121.

[4] H. S. J. van der Zant, W. J. Elion, L. J. Geerligs, and J. E. Mooij, Phys. Rev. B 54, 10081 (1996).

[5] R. Fazio and G. Schön, Phys. Rev. B 43, 5307 (1991).

[6] A. A. Middleton and N. S. Wingreen, Phys. Rev. Lett. 71, 3198 (1993); A. D. Zaikin and S. V. Panyukov, cond-mat/ 9510159.

[7] E. Granato and J. M. Kosterlitz, Phys. Rev. B 33, 6533 (1986); Phys. Rev. Lett. 62, 823 (1989).

[8] M. P. Fisher, T. A. Tokuyasu, and A. P. Young, Phys. Rev. Lett. 66, 2931 (1991); R. A. Hyman, M. Wallin, M. P. A. Fisher, S. M. Girvin, and A.P. Young, Phys. Rev. B 51, 15304 (1995).

[9] J. Kogut, Rev. Mod. Phys. 51, 659 (1979).

[10] This is the analogous effect of an applied current for vortex motion in superconductors [8].

[11] S. Teitel and C. Jayaprakash, Phys. Rev. Lett. 51, 1999 (1983).

[12] J. M. Thijssen and H. J.F. Knops, Phys. Rev. B 37, 7738 (1988).

[13] K. A. Benedict and M. A. Moore, Phys. Rev. B 39, 4592 (1989).

[14] U. Geigenmüller and G. Schön, Europhys. Lett. 10, 765 (1989). 